\title{
ORIGINAL
}

\section{TRATAMIENTO ESPECIALIZADO DEL ABUSO O DEPENDENCIA DEL ALCOHOL (*)}

Josep M Suelves (1), Joan R Villalbí $(2,3,4,5)$, Marina Bosque-Prous $(2,4,5)$, Albert Espelt $(2,3.4,6)$ y M Teresa Brugal $(2,4)$.

(1) Agència de Salut Pública de Catalunya.

(2) Agència de Salut Pública de Barcelona.

(3) CIBER de Epidemiología y Salud Pública.

(4) Institut d'Investigació Biomèdica Sant Pau (IIB Sant Pau).

(5) Departament de Ciències Experimentals i de la Salut, Universitat Pompeu Fabra.

(6) Departament de Psicobiologia i Metodologia de les Ciències de la Salut. Universitat Autònoma de Barcelona.

(*) Este trabajo se ha beneficiado de la financiación parcial de las Redes de investigación cooperativa (Red trastornos adictivos RTA) RD06/0001/1018 y RD12/0028/0018. Se ha realizado en el marco del Grupo de Trabajo sobre Alcohol de la Sociedad Española de Epidemiología. Los autores agradecen la ayuda económica del Plan Nacional sobre Drogas que ha contribuido a su funcionamiento (órdenes SSI $2800 / 2012$ y $131 / 2013)$.

Los autores declaran no tener ningún conflicto de intereses.

\section{RESUMEN}

Fundamentos. El Plan Nacional sobre Drogas (PNSD) impulsó la prevención y el tratamiento de los trastornos por uso de drogas ilegales, y ahora abarca los trastornos por uso de alcohol. El objetivo de este trabajo es realizar una estimación de las personas en tratamiento especializado por alcohol en la red pública.

Métodos. Se revisan documentos del PNSD, del sistema de información sobre drogodependencias de Cataluña y del de Barcelona. Para Cataluña y Barcelona se presentan datos de 1991 a 2010.

Resultados. Para el conjunto de España hay información disponible desde hace pocos años, con exhaustividad y validez variable. En Catalunya, el número de admisiones a tratamiento por alcohol notificadas se incrementó: el alcohol causa anualmente más del $40 \%$ del total de admisiones a tratamiento en la red de drogodependencias; la edad media es de 44 años y el $23 \%$ son mujeres. En Barcelona las admisiones a tratamiento por alcohol superan las 2.000 al año, suponiendo 217 por 100.000 habitantes mayores de 15 años varones y 67 en mujeres.

Conclusiones. Los datos disponibles sugieren que por lo que respecta al alcohol el sistema de información sobre drogas ha de desarrollarse más en España, resolviendo problemas metodológicos. Los datos disponibles para Cataluña sugieren que la red de atención a drogas ha permitido abordar la necesidad asistencial por dependencia al alcohol conjuntamente con la de drogas ilegales. Esta información es relevante, pues apenas existen datos de tratamiento referidos a la población.

Palabras clave. Abuso del alcohol. Dependencia del alcohol. Tratamiento por alcohol. Sistemas de información. Investigación sobre servicios sanitarios.

Correspondencia

Josep M Suelves

Agència de Salut Pública de Catalunya

C/ Roc Boronat 81

08005 Barcelona

josepmaria.suelves@gencat.cat

\section{ABSTRACT \\ Specialised Treatment for Alcohol Abuse or Dependence}

Background. The National Plan for Drugs in Spain (PNSD) fostered the prevention and treatment of illegal drug use disorders, and now also covers alcohol use disorders. The goal of this paper is to estimate the number of persons in specialized treatment because of alcohol in public services.

Methods. Review of the key documents of the PNSD, the drug information system of Catalonia, and the Barcelona drug information system. For Catalonia and Barcelona data from 1991 to 2010 are presented.

Results. In recent years, there has been more information available for Spain as a whole, of varying validity and comprehensiveness. In Catalonia, the number of reported admissions to treatment for alcohol use disorders has risen: alcohol causes yearly over $40 \%$ of all admissions in the drug addiction treatment network; mean age is 44 years, and $23 \%$ are women. In Barcelona, admissions to treatment due to alcohol are more than 2.000 each year, relative frequency is 217 by 100.000 residents over 15 years for men and 67 for women.

Conclusions. Available data shows that for alcohol, the drug information system needs further development in Spain, solving methodological issues. Available data for Catalonia suggests that the network of drug treatment centres has improved treatment for alcohol use disorders along with that of illegal drugs. This information is relevant, as there is almost no population based treatment data. This information should be completed for other Regional Administrations.

Key words. Alcohol abuse. Alcohol dependence. Alcohol treatment. Information systems. Health service research. 


\section{INTRODUCCIÓN}

El desarrollo del Plan Nacional sobre Drogas (PNSD), existente desde 1985, comportó un impulso considerable a las políticas de prevención y tratamiento de los trastornos por uso de drogas ilegales ${ }^{1}$. El PNSD se configuró como un órgano de coordinación dependiente del gobierno (con mayor vinculación al Ministerio de Sanidad o al de Interior, según el periodo), con la función de armonizar, mediante el consenso y la distribución de recursos económicos, las políticas sectoriales sobre drogas de las comunidades autónomas (CCAA) que tienen las competencias en materia de educación, servicios sociales y salud.

El PNSD, inicialmente centrado en los graves problemas que el consumo de heroína causaba, fue incorporando objetivos vinculados a la prevención del uso de drogas y sus complicaciones y desde hace algunos años también incluye el abordaje de los trastornos por uso de alcohol ${ }^{2}$. Aunque muchas CCAA incluyen el alcohol en su planificación en materia de prevención y tratamiento de los trastornos por uso de drogas, el hecho de que no formara parte de las estrategias iniciales del PNSD puede haber contribuido a una mayor diversidad en las políticas de salud en relación al alcohol de la que se observa en relación a las drogas ilegales y a que no se haya analizado el acervo de datos existente de forma sistemática para extraer la información que proporcionan.

El objetivo del presente trabajo fue realizar una estimación del número de personas que realizaron en tratamiento especializado por trastornos por uso de alcohol en la red pública en España.

\section{MATERIAL Y MÉTODOS}

Fuentes de información. Procede de las Memorias anuales ${ }^{3}$ y los informes del
Observatorio Español sobre Drogas (OED) del PNSD ${ }^{4}$. Las memorias del PNSD incluyen información proporcionada por las CCAA sobre la atención por problemas de alcoholismo, con datos sobre oferta de tratamiento y usuarios. Por otra parte, los informes del OED contienen información sobre las admisiones a tratamiento por consumo alcohol extraída de uno de los indicadores clave del sistema de información sobre toxicomanías que se puso en marcha en $1987^{5}$. Éste incluyó inicialmente las admisiones a tratamiento por abuso y dependencia de los opiáceos y la cocaína en centros especializados. En 1991 se amplió a todas las drogas ilegales y más tarde al alcohol ${ }^{6}$. Los informes basados en este sistema de registro gestionado por las comunidades autónomas, que controlan que no exista más de un registro anual por individuo, y que se compilan en el PNSD, han empezado recientemente a hacer públicos sus datos sobre alcohol, aunque con poca desagregación. En el momento de redactar este trabajo estaba disponible la Memoria del PNSD del año 2009 y el informe del Observatorio de 2011, con datos referidos también a 2009 para las admisiones a tratamiento.

La Agència de Salut Pública de Catalunya mantiene un sistema de información sobre drogodependencias (SID) que recoge la actividad asistencial de los más de 60 Centros de Atención y Seguimiento (CAS) que forman parte de la red de atención a las drogodependencias de financiación pública en Cataluña. Este sistema, que desde 1987 recoge las admisiones a tratamiento especializado ambulatorio del abuso y la dependencia del alcohol en la misma base de datos que registra las admisiones a tratamiento por drogas ilegales, está accesible a través de internet y genera informes periódicos sistemáticos desde $1991^{7}$. Los datos sobre admisiones a tratamiento de este sistema son los que se remiten al OED para aportar la infor- 
mación de Cataluña. Se incluyen los datos desde 1991 (cuando se amplió notablemente la oferta de tratamiento de mantenimiento con metadona y el sistema de información se estabilizó) hasta 2010.

Para la ciudad de Barcelona los datos se extraen del Sistema de Información sobre Drogas de la ciudad de Barcelona (SIDB), que recoge información sobre pacientes y tratamientos en los centros de la red de atención a las drogodependencias de financiación pública en la ciudad, que a su vez alimenta el SID de Cataluña ${ }^{8}$. En la ciudad, esta red en 2010 estaba compuesta por 16 centros ambulatorios de los que 4 están en los hospitales universitarios que estructuran las cuatro áreas territoriales de atención especializada en la ciudad. De los 16 centros, 2 son monográficos para pacientes con problemas de alcohol, 1 es un recurso de baja exigencia que se concentra en atraer usuarios activos de drogas ilegales y los otros 13 acogen a usuarios de diversas sustancias. El sistema identifica admisiones a tratamiento (que puede ser en pacientes tratados previamente si han pasado 6 meses desde que abandonaron el tratamiento) y pacientes en tratamiento. Anualmente se inician unos 5.000 tratamientos por todas las sustancias $^{9}$. El patrón asistencial muestra una clara estacionalidad con más admisiones en invierno y menos en verano. La red es relativamente estable. Al igual que para Cataluña, se incluyen los datos a partir de 1991 (cuando se amplió notablemente la oferta de tratamiento de mantenimiento con metadona y el sistema de información se estabilizó) hasta 2010.

\section{RESULTADOS}

Los datos de centros de tratamiento y usuarios de los mismos notificados por las CCAA al PNSD y recogidos en su Memoria anual para 2009 se presentan en las primeras columnas de la tabla 1 . Como puede verse, todas las CCAA disponen de oferta asistencial. Para poder comparar los usuarios en términos relativos se ha estimado su frecuencia por 100.000 habitantes mayores de 15 años. Las cifras presentan importantes variaciones, siendo máximas en Aragón y Asturias, seguidas por Baleares, Extremadura y el País Vasco.

Con respecto a las admisiones a tratamiento en España presentadas en el informe del OED, en 2009 todas las comunidades autónomas declararon casos. Aquel año, el alcohol representó el 34,7\% de todos las admisiones a tratamiento notificadas al PNSD por las comunidades autónomas (27.954 casos de un total de 80.503 admisiones a tratamiento), cifra que supone un fuerte incremento con respecto a los datos de años antes. No hay datos accesibles desglosados por CCAA.

La figura 1 muestra como en Cataluña, el número de admisiones a tratamiento por alcohol notificadas anualmente siguió una tendencia ascendente, moderándose a partir de 2003. Los tratamientos por alcohol representaron en 2010 el 45,7\% del total del total de tratamientos que se inician en la red de atención a drogodependencias. Los datos del último año permiten apreciar que el $77 \%$ eran hombres y el $23 \%$ mujeres. La edad media de los casos era de 44 años. Un $45 \%$ habían sido derivados desde otros centros sanitarios: el $32,7 \%$ de los casos por un centro de atención primaria de salud y un $12,8 \%$ por otros servicios sanitarios. Otros rasgos destacables son que el $11,0 \%$ de los usuarios atendidos no había completado los estudios primarios y el $41,0 \%$ estaba en paro.

En Barcelona se registró un incremento de las admisiones a tratamiento por consumo de alcohol mientras que la suma de admisiones a tratamiento por las tres drogas ilegales más importantes (heroína, cocaína y cannabis) se mantuvo estable e, incluso, disminuyó discretamente, como 
Tabla 1

Centros ambulatorios de atención a problemas de alcoholismo y usuarios de los mismos notificados al Plan Nacional sobre Drogas por Comunidades Autónomas. España, 2009

\begin{tabular}{|c|c|c|c|c|}
\hline Comunidad autónoma & Centros & Usuarios & Población $>$ de 15 años & Usuarios por 100.000 habitantes \\
\hline Andalucía & 119 & 6.346 & 6.836 .280 & 92,82 \\
\hline Aragón & 16 & 2.913 & 1.142 .906 & 254,87 \\
\hline Asturias & 18 & 2.613 & 946.724 & 276,00 \\
\hline Baleares & 14 & 2.217 & 904.924 & 244,99 \\
\hline Canarias & 31 & 2.168 & 1.763 .480 & 122,93 \\
\hline Cantabria & 3 & 63 & 504.277 & 12,49 \\
\hline Castilla la Mancha & 9 & 1.080 & 1.733 .129 & 62,31 \\
\hline Castilla y León & 52 & 3.405 & 2.214 .479 & 153,76 \\
\hline Catalunya & 62 & 6.603 & 6.175 .473 & 106,92 \\
\hline Extremadura & 28 & 2.197 & 923.424 & 237,91 \\
\hline Galicia & 6 & 2.181 & 2.421 .721 & 90,05 \\
\hline Madrid & 39 & Sin datos & 5.319 .732 & Sin datos \\
\hline Murcia & 10 & 1.795 & 1.199 .133 & 149,69 \\
\hline Navarra & 14 & 1.025 & 524.509 & 195,42 \\
\hline País Vasco & 36 & 4.440 & 1.857 .623 & 239,01 \\
\hline La Rioja & 7 & 346 & 272.063 & 127,17 \\
\hline Valencia & 42 & 4.509 & 4.261 .224 & 105,81 \\
\hline
\end{tabular}

Fuente: Plan Nacional Sobre Drogas. Memoria anual 2009.

\section{Figura 1}

Personas admitidas a tratamiento en los Centros de Atención y Seguimiento (CAS) de la red de atención a drogodependencias de financiación pública, según sustancia principal que motiva la demanda de tratamiento. Cataluña, 1991-2010

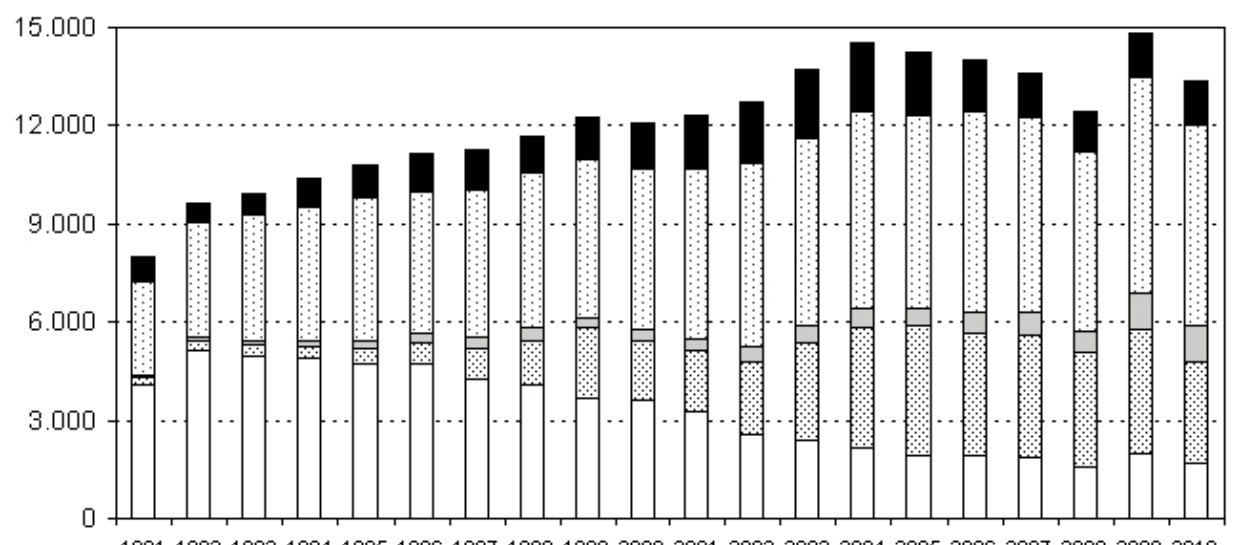

$\square$ Heroina . O Cocaina $\square$ Cannabis $\square$ Alcohol a Otros 
se aprecia en la figura 2. En los últimos años las admisiones a tratamiento por alcohol oscilaron alrededor de 2.200 al año, lo que representaba el $42 \%$ de las admisiones por todas las sustancias. Del total de inicios de tratamiento por trastornos por consumo de alcohol, el 75\% correspondió a varones y el $25 \%$ a mujeres.

Con los datos de los pacientes residentes en Barcelona (que eran el 91\% de los atendidos en la ciudad), se estimaron tasas anuales de admisión a tratamiento por edad y sexo (figura 3). La tasa anual de admisiones por 100.000 habitantes mayores de 15 años fue de 217 en varones y 67 en mujeres. En los grupos de edad de 35-44 y 45-54 años, que fueron los que tuvieron más casos, alcanzaron tasas de $300-400$ por 100.000 personas año en varones y de 100-150 en mujeres. Si se observan las admisiones a tratamiento según lugar de residencia, oscilaron de forma importante (datos no mostrados procedentes del SIDB): las diferencias parecen relacionadas con el nivel socioeco- nómico y podrían reflejar un menor uso de la red pública en los distritos de mayor renta por habitante.

Se dispone de datos más exhaustivos para aquellos centros de Barcelona que comparten componentes del sistema de información propio de la Agència de Salut Pública de Barcelona (que cubren el 62\% de los tratamientos declarados en la ciudad). En estos centros se constató que cada paciente en tratamiento con alcohol generó una media de 28 visitas/año y que la razón entre el número de pacientes en tratamiento y la cifra de primeras visitas fue de 2 a 1 (algo superior en mujeres, que presentan mayor adherencia al tratamiento). En estos centros, los pacientes en programa de alcohol fueron el $40 \%$ de las nuevas admisiones a tratamiento, pero representaron un tercio del total de pacientes atendidos en el centro y realizaron un tercio del total de visitas. Cada año el 5\% de los pacientes atendidos recibió el alta terapéutica.

Figura 2

Personas admitidas a tratamiento en los Centros de Atención y Seguimiento (CAS) de la red de atención a drogodependencias de financiación pública, según sustancia principal que motiva la demanda de tratamiento. Barcelona, 1991-2010.

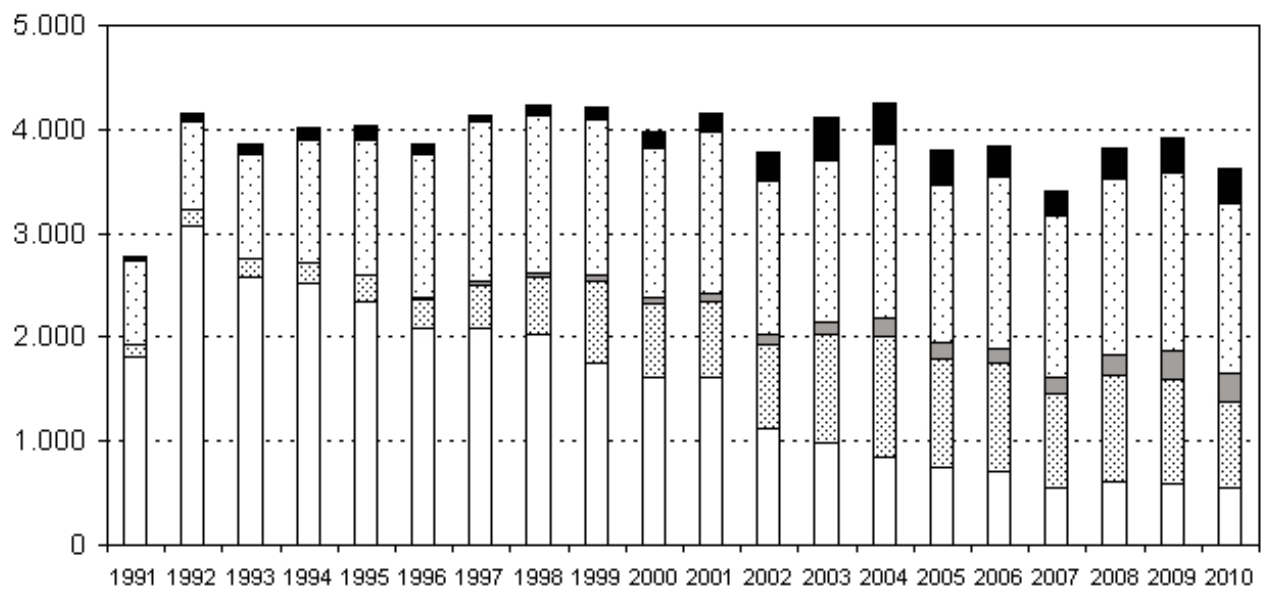

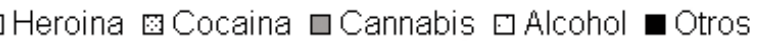


Figura 3

Admisiones a tratamiento por trastornos por consumo de alcohol en personas residentes en la ciudad (por 100.000 habitantes mayores de 15 años) en los Centros de Atención y Seguimiento (CAS) de la red de atención a drogodependencias de financiación pública, por grupos de edad y sexo. Barcelona, 1991-2010.
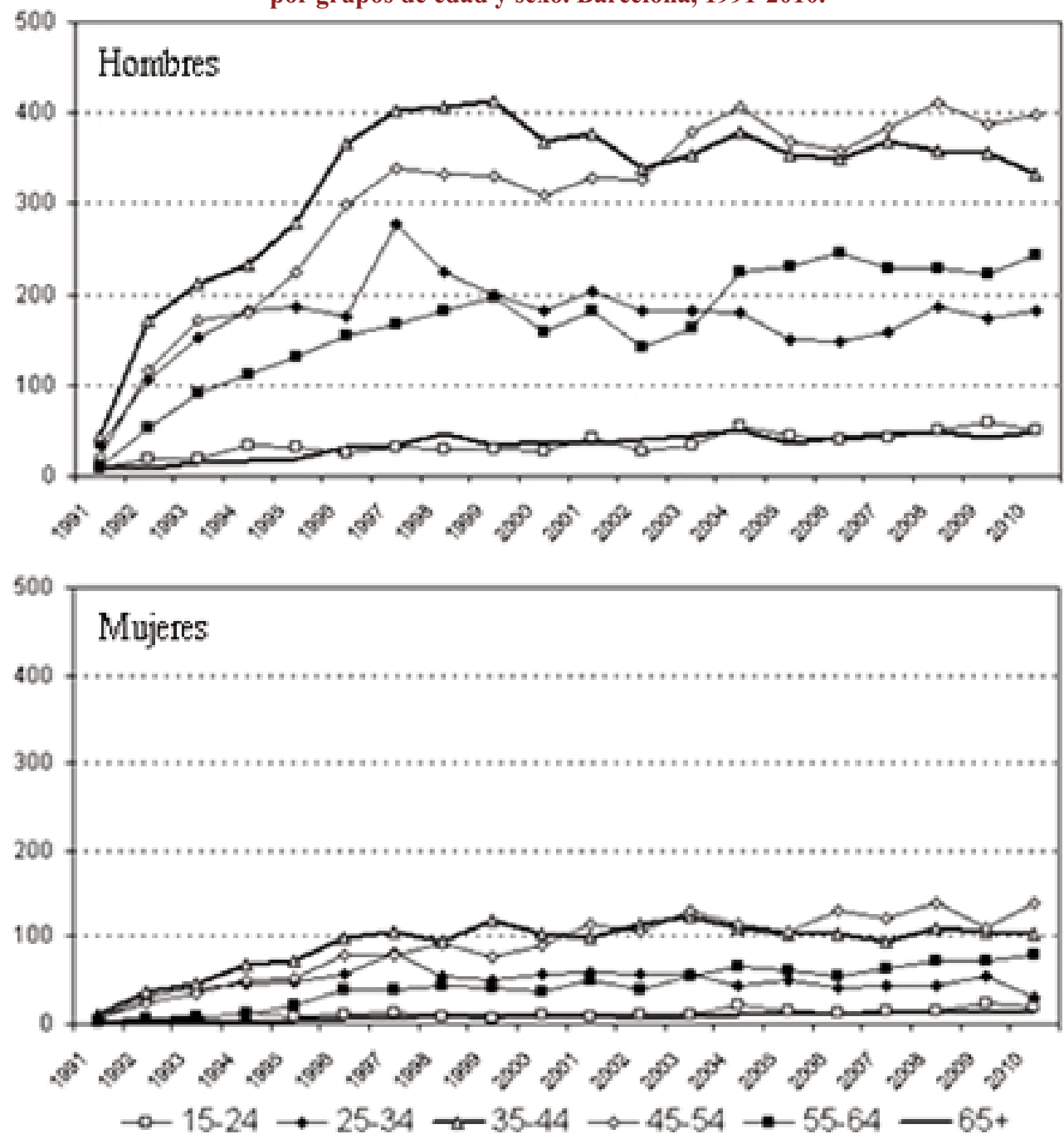

\section{DISCUSION}

Las cifras para el conjunto de España sugieren que los datos sobre tratamiento por alcohol que el PNSD publica anualmente no están totalmente consolidados como sistema de información estable. Las cifras incluidas en las memorias no parecen responder a criterios comunes en su recogida y probablemente tienen significados diversos en distintas CCAA. Es probable que, a diferencia de las drogas ilegales, no haya una homogeneización de criterio sobre lo que se considera inicio de tratamiento por alcohol. 
Así, algunas notifican exclusivamente el número de admisiones a tratamiento mientras que otras informan sobre el total de personas tratadas anualmente o sobre contactos con servicios asistenciales que no siempre comportan un tratamiento de los trastornos por uso de alcohol en sentido estricto. Por lo que respecta a las admisiones de tratamiento notificadas al OED, podría ser que algunas CCAA incluyeran datos de fuentes diversas, mientras que en otras los datos facilitados se limitaran a los centros de la red pública de atención a drogas. Al derivar de sistemas de información más orientados a un uso administrativo que sanitario, es probable que actualmente algunas CCAA incluyan tanto tratamientos específicos de los trastornos por uso de alcohol como casos con un consumo de riesgo que reciben un consejo breve desde los servicios de atención primaria de salud, casos tratados por otros trastornos de salud mental con un diagnóstico asociado de trastorno por uso de alcohol o personas atendidas por organizaciones de autoayuda subvencionadas sin que exista un diagnóstico clínico.

En cualquier caso, es razonable esperar un crecimiento de la calidad y la exhaustividad de los datos que, probablemente, ya sean elevadas en algunas CCAA, convirtiéndose por tanto en una información con gran potencial para estudios e investigaciones ${ }^{10}$. Un análisis restringido a las CCAA que proporcionan datos más homogéneos ofrecería información de gran interés mientras el sistema de información acaba de desarrollarse en el conjunto de España. En cualquier caso, las admisiones notificadas en relación a la población mayor de 15 años de estos territorios permitiría estimar un mínimo de 72 admisiones a tratamiento especializado por 100.000 personas año.

Las admisiones a tratamiento por alcohol en centros especializados no suponen la única fuente de información sobre atención a las personas con trastornos por con- sumo de alcohol. En Cataluña, los hospitales de agudos notificaron en 2010 un total de 1.297 contactos de hospitalización por trastornos relacionados con el consumo de alcohol (edad media 46 años, $23 \%$ mujeres $)^{11}$. Según el mismo informe, los hospitales monográficos psiquiátricos notificaron ese mismo año 562 ingresos por síndrome de dependencia del alcohol (edad media 44 años, $27 \%$ de mujeres) y las unidades de psiquiatría de hospitales generales 728 casos más (46 años, 23\% mujeres). Finalmente, los centros de salud mental de adultos declararon 1.084 episodios (edad media 48,1, siendo un 26,9\% mujeres). Estos datos se derivan del Conjunto Mínimo Básico de Datos (CMDB), un sistema de información sanitario poblacional que incluye hospitales y centros ambulatorios de salud mental, pero que no incluye los centros de la red de atención a drogodependencias, salvo aquellos que forman también parte de la red de atención en salud mental.

La evolución de las admisiones a tratamiento en Barcelona y Cataluña muestra cómo la consolidación de la red de atención a drogas en esta comunidad ha permitido mejorar el abordaje de las necesidades asistenciales por abuso y dependencia del alcohol, habiendo partido hace 20 años de cifras muy bajas. La reducción de la demanda de nuevos tratamientos por opiáceos a partir de finales de los años 1990 pudo haber facilitado el incremento de la oferta asistencial para los pacientes con dependencia al alcohol. Aunque actualmente parece haberse alcanzado un equilibrio en la demanda de tratamiento. Esto es especialmente visible en la ciudad de Barcelona, donde el peso de la heroína en la demanda de tratamiento era muy importante a principios de los años 90, y el número de admisiones a tratamiento por drogas ilegales ha disminuido en términos absolutos, en contraste con las motivadas por el alcohol. Hay que tener en cuenta que la red de atención primaria 
en Cataluña está aplicando protocolos estandarizados para atender a un número creciente de bebedores de riesgo que no presentan criterios de abuso o dependencia $^{12}$. Además, hay cierta oferta de atención privada al margen de la red de financiación pública y existe una red más informal de grupos de ayuda mutua que opera al margen del sistema sanitario (destacando el rol de la asociación Alcohólicos Anónimos).

Los datos observados sugieren que las personas con un trastorno por consumo de alcohol pueden contar hoy día con una oferta de tratamiento que años atrás era muy inferior en términos poblacionales. De todas formas, las cifras se muestran bastante inferiores a las de países como Francia (2.450 usuarios por 100.000 habitantes de 15 o más años en los centros de atención ambulatoria en alcohología ${ }^{13} \mathrm{o}$ los EEUU (282 admisiones a tratamiento por 100.000 personas de 12 o más años en 2010 en centros de tratamiento licenciados o certificados por los estados) ${ }^{14}$. Para Inglaterra, los datos publicados de atención a los problemas de alcohol en 201112 son algo superiores a los correspondientes a la atención especializada en Barcelona, aunque incluyen los casos gestionados por el médico de cabecera ${ }^{15}$. Estos datos muestran también el predominio de varones y de los grupos de edad de 35-54 años en la demanda de tratamiento por problemas con el consumo de alcohol.

Lo cierto es que cuesta encontrar datos publicados de tratamiento referidos a la población, tanto en España como internacionalmente $^{16}$. Los datos para toda España recogidos en el informe del Observatorio Español de la Droga y las Toxicomanías del año 2011 muestran un patrón similar por edad y sexo, pero si se estiman tasas de admisión por 100.000 habitantes son muy inferiores a las de Barcelona ${ }^{17}$. Esta información se debe enmarcar con las estimaciones poblacionales de necesidad de abor- daje, como las de prevalencia de abuso o dependencia del alcohol realizadas en la encuesta ESEMED ${ }^{18}$ o las estimaciones de consumo de riesgo de $11-18 \%$ en la población general derivadas de cribados en atención primaria ${ }^{19,20}$.

\section{BIBLIOGRAFÍA}

1. Torres Hernández MA (ed). Historia de las adicciones en la España contemporánea. Barcelona: Socidrogalcohol; 2009.

2. Álvarez E, Brime B, González J, Llorens N, Ruiz A, Sendino R. Observatorio Español de la droga y las toxicomanías: informe 2011. Madrid: Ministerio de Sanidad, Política Social e Igualdad; 2011.

3. Publicaciones de la Delegación del Gobierno para el Plan Nacional sobre Drogas. Disponible en: http://www.pnsd.msssi.gob.es/Categoria2/publica/publicaciones/home.htm

4. Informes del Observatorio Español sobre Drogas. Disponible en: http://www.pnsd.msssi.gob.es/Categoria2/observa/oed/home.htm

5. Alvarez-Requejo A, Suelves JM, Brugal MT, Correa JF. Monitoring treatment demand for drug abuse in Spain: perspective over a decade. Eur Addict Res. 1999; 5: 179-84.

6. Suelves JM. Drogodependencias: de la epidemiología a la intervención. Estrategias y oportunidades. Rev Esp Drogodependencias. 2005; 30: 231-42

7. Sistema d'Informació sobre Drogues de Catalunya. http://www20.gencat.cat/portal/site/canalsalut/menuitem.dda7170e89086516ba963bb4b0c0e1a0/?vgnextoid $=0714 \mathrm{a} 347 \mathrm{f} 6164310 \mathrm{VgnVCM} 1000008 \mathrm{~d} 0 \mathrm{c} 1 \mathrm{e} 0 \mathrm{aR}$ CRD\&vgnextchannel $=0714 \mathrm{a} 347 \mathrm{f} 6164310 \mathrm{VgnVCM} 1$ 000008d0c1e0aRCRD\&vgnextfmt=default

8. Servei d'Atenció i Prevenció de les Drogodependències. Sistema d'Informació sobre Drogues de Barcelona (SIDB). Indicadors corresponents al segon i tercer trimestre de 2012. Barcelona: Agència de Salut Pública de Barcelona; 2013. Disponible en http://www.aspb.cat.

9. Brugal MT, Guitart A, Espelt A. Pla d'Acció Sobre Drogues de Barcelona 2013-16. Barcelona: Agència de Salut Pública de Barcelona; 2013.

10. Evans E, Grella CE, Murphy DA, Hser YI. Using administrative data for longitudinal substance abuse research. J Behav Health Serv Res. 2010; 37: 252-71. 
11. Divisió de Registres de Demanda i Activitat. Activitat assistencial de la xarxa sanitària de Catalunya. Any 2010. Registre del conjunt mínim bàsic de dades (CMDB). Barcelona: Servei Català de la Salut, 2012. Disponible en http://www20.gencat.cat/docs/canalsalut/Minisite/ObservatoriSalut/ossc Dades estadistiques/Sistema_sanitari/Activitat/Cmbd/Fitxers_estatics/A ctivitat_xarxa_assistencial_cmbd_2010.pdf

12. Aubà Llambrich J, Freixedas Casaponsa R. La detección del consumo de alcohol en atención primaria. Aten Primaria. 2000; 25: 144-51.

13. Fédération Nationale dels Observatoires Régionaux de Santé. Les addictions dans les régions de France. Paris: Fédération Nationale dels Observatoires Régionaux de Santé \& Ministère de la Santé, de la Jeunesse et des Sports; 2007.

14. Substance Abuse and Mental Health Services Administration, Center for Behavioral Health Statistics and Quality. Treatment Episode Data Set (TEDS): 2000-2010. State Admissions to Substance Abuse Treatment Services, DASIS Series: S-63, HHS Publication No. SMA-12-4729. Rockville, MD: Substance Abuse and Mental Health Services Administration; 2012

15. Statistics for alcohol treatment activity in England 2011-12. National Drug Treatment Monitoring System. London: The National Treatment Agency for Substance Misuse; 2013.

16. Wolstenholme A, Drummond C, Deluca P, Davey $Z$, Elzerbi $C$, Gual A et al. Alcohol interventions and treatments in Europe. En: Anderson P, Braddick F, Reynolds J, Gual A (Eds). Alcohol policy in Europe: evidence from AMPHORA. The AMPHORA project, 2012; 55-80. Disponible en http://www.amphoraproject.net/view.php?id_cont $=32$

17. Observatorio Español de la Droga y las Toxicomanías. Informe 2011. Madrid: Delegación del Gobierno para el Plan Nacional sobre Drogas; 2012.

18. Haro JM, Palacín C, Vilagut G, Martínez M, Bernal M, Luque I et al. Prevalencia de los trastornos mentales y factores asociados. El estudio ESEMeD-España. Med Clin (Barc). 2006; 126: 445-51.

19, López-Marina V, Pizarro Romero G, Alcolea García R, Beato Fernández P, Galindo Montané E, Montellá Jordana N. Evaluación del cribado y la efectividad de una intervención breve en bebedores de riesgo atendidos en consultas de atención primaria. Aten Primaria. 2005; 36: 261-8.
20. Segura García L, Gual Solé A, Montserrat Mestre O, Bueno Belmonte A, Colom Farran J. Detección y abordaje de los problemas de alcohol en la atención primaria de Cataluña. Aten Primaria 2006; 37: 484-90. 